\title{
Comunicación
}

\section{Determinación del Perfil Bioquímico Sanguíneo Hepático y Renal en Alpacas (Vicugna pacos) Aparentemente Normales}

\author{
Determination of Blood Biochemical Profile of Liver and Kidneys in Healthy \\ Alpacas (Vicugna pacos)
}

\author{
Sergio Flores N. ${ }^{1,3}$, Olga Li E. ${ }^{1,4}$, César Gavidia C. ${ }^{2}$, Luis Hoyos S. ${ }^{1}$, \\ Manuel Barrios-Arpi ${ }^{1}$
}

\section{Resumen}

\begin{abstract}
El objetivo del presente estudio fue determinar el perfil bioquímico hepático y renal en alpacas (Vicugna pacos) aparentemente normales de un rebaño de la Sierra Central del Perú. Se tomaron muestras de sangre $(8 \mathrm{ml})$ en 60 animales ( 30 adultos y 30 tuis), mediante punción de la vena yugular. Los valores promedio fueron Bilirrubina total: $0.62 \pm 0.51 \mathrm{mg} / \mathrm{dl}$; Bilirrubina directa: $0.13 \pm 0.09 \mathrm{mg} / \mathrm{dl}$; Bilirrubina indirecta: $0.51 \pm 0.52 \mathrm{mg} / \mathrm{dl}$; ALT: $23.27 \pm$ 13.11 UI/L; AST: 197.2 \pm 53.74 UI/L; Fosfatasa alcalina: $159.45 \pm 76.6$ UI/L; GGT: $22.35 \pm$ 10.63; Proteínas totales: $7.73 \pm 1.18 \mathrm{~g} / \mathrm{dl}$; Albúmina: $3.63 \pm 0.65 \mathrm{~g} / \mathrm{dl}$; Globulina: $4.10 \pm$ $1.40 \mathrm{~g} / \mathrm{dl}$; Urea: $39.1 \pm 9.02 \mathrm{mg} / \mathrm{dl}$ y Creatinina: $2.21 \pm 0.54 \mathrm{mg} / \mathrm{dl}$. Se encontró diferencia estadística $(\mathrm{p}<0.05)$ entre grupos etarios para bilirrubina total e indirecta y fosfatasa alcalina.
\end{abstract}

Palabras clave: Vicugna pacos, tuis, bioquímica sanguínea

\section{Abstract}

The aim of this study was to establish the blood biochemical profile of liver and kidney in clinically healthy alpacas (Vicugna pacos) of the central highlands of Peru. Blood samples ( $8 \mathrm{ml}$ ) were collected from 60 animals ( 30 adults and 30 tuis) by puncture of the jugular vein. The mean values were Total bilirubin: $0.62 \pm 0.51 \mathrm{mg} / \mathrm{dl}$; Direct bilirubin: $0.13 \pm 0.09 \mathrm{mg} / \mathrm{dl}$; Indirect bilirubin: $0.51 \pm 0.52 \mathrm{mg} / \mathrm{dl}$; ALT: $23.27 \pm 13.11 \mathrm{IU} / \mathrm{L} ;$ AST: 197.2

\footnotetext{
${ }^{1}$ Laboratorio de Patología Clínica y Biología Molecular, ${ }^{2}$ Laboratorio de Medicina Preventiva, Facultad de Medicina Veterinaria, Universidad Nacional Mayor de San Marcos, Lima, Perú

${ }^{3}$ E-mail: anflav@hotmail.com

${ }^{4}$ E-mail: olgalie@hotmail.com
}

Recibido: 6 de junio de 2015

Aceptado para publicación: 17 de setiembre de 2015 
\pm 53.74 IU; Alkaline phosphatase: $159.45 \pm 76.6 \mathrm{IU} / \mathrm{L}$; GGT: $22.35 \pm 10.63$; Total protein: $7.73 \pm 1.18 \mathrm{~g} / \mathrm{dl}$; Albumin: $3.63 \pm 0.65 \mathrm{~g} / \mathrm{dl}$; Globulin: $4.10 \pm 1.40 \mathrm{~g} / \mathrm{dl}$; Urea: $39.1 \pm 9.02 \mathrm{mg} / \mathrm{dl}$; and Creatinine: $2.21 \pm 0.54 \mathrm{mg} / \mathrm{dl}$. Statistical difference was found $(\mathrm{p}<0.05)$ between age groups for total and indirect bilirubin and alkaline phosphatase.

Key words: Vicugna pacos, tuis, blood biochemistry

\section{INTRODUCCIÓN}

El Perú posee el $87 \%$ de las alpacas a nivel mundial. La mayoría de ellas son criadas y manejadas por miles de familias campesinas que forman parte de los sectores más pobres del país. Asimismo, es una especie clave dentro de la ecología y es socio-económicamente de gran valor para el poblador rural de la región andina.

Se han reportado estudios sobre parámetros bioquímicos en camélidos sudamericanos, tanto en buena condición de salud como en enfermedad (Anderson, 2002); sin embargo, existe escasa información sobre la bioquímica sanguínea de la alpaca en diferentes etapas de su vida productiva, y la mayoría no exhibe el perfil hepático, renal, pancreático o cardiaco de alpacas o llamas criadas en sus hábitats naturales (Concha, 2009).

Los trabajos existentes están mayormente relacionados a los valores normales de los componentes bioquímicos de los líquidos orgánicos de la alpaca. Trabajos realizados en Europa, Norteamérica y de la zona andina han empleado mayormente un reducido número de animales o limitados a variables hematológicas o bioquímicas (Lassen et al., 1986; Fowler, 1989; Garry, 1989; ISIS, 1999; Concha, 2009). Es así, que el presente estudio tuvo por objetivo la determinación de los valores bioquímicos hepáticos y bioquímicos renales en dos grupos etarios de alpacas.

\section{Materiales y Métodos}

El estudio se realizó en noviembre de 2007 en la SAIS Túpac Amaru, localizada en los poblados de Cochas y Pachacayo, distrito de Jauja, departamento de Junín, a una altitud de 3970 msnm. La temperatura ambiental promedio en la zona fue de $12.6^{\circ} \mathrm{C}$ y con una humedad relativa media entre 45 y $53 \%$ en los días del muestreo (SENAMHI, 2009).

Se utilizaron 60 animales (30 adultos y 30 tuis) aparentemente saludables. La alimentación de los animales fue sobre pasturas naturales propias de la zona y con agua administrada ad libitum. El número de animales fue calculado según el teorema de límite central (Daniel, 1996).

Se recolectaron muestras de sangre en horas de la mañana. La sangre $(8 \mathrm{ml})$ fue extraída por punción de la vena yugular en tubos sin anticoagulante y por medio de aguja descartable 21G. Los sueros fueron extraídos por decantación luego de la formación del coágulo y fueron trasladados en cajas térmicas con bolsas refrigerantes al Laboratorio de Patología Clínica y Biología Molecular de la Facultad de Medicina Veterinaria, Universidad Nacional Mayor de San Marcos, Lima. En el laboratorio se volvieron a centrifugar a $1300 \mathrm{~g}$ durante 10 minutos.

Se determinaron los valores séricos de bilirrubina, proteínas totales, albúmina, urea y creatinina por métodos colorimétricos, y las enzimas alanina transaminasa (ALT), 
Cuadro 1. Perfil bioquímico sanguíneo hepático en 60 alpacas (Vicugna pacos) de la Sierra central del Perú

\begin{tabular}{lcccc}
\hline Parámetro & Media & d.e. & Mínimo & Máximo \\
\hline Bilirrubina total $(\mathrm{mg} / \mathrm{dl})$ & 0.62 & 0.51 & 0.10 & 2.30 \\
Bilirrubina directa $(\mathrm{mg} / \mathrm{dl})$ & 0.13 & 0.09 & 0.01 & 0.50 \\
Bilirrubina indirecta $(\mathrm{mg} / \mathrm{dl})$ & 0.51 & 0.52 & 0.05 & 2.25 \\
Proteínas totales $(\mathrm{g} / \mathrm{dl})$ & 7.73 & 1.18 & 6.00 & 12.20 \\
Albúmina $(\mathrm{g} / \mathrm{dl})$ & 3.63 & 0.65 & 2.60 & 5.40 \\
Globulina $(\mathrm{g} / \mathrm{dl})$ & 4.10 & 1.40 & 1.20 & 9.40 \\
ALT (UI/L) & 23.27 & 13.11 & 5.00 & 80.00 \\
AST (UI/L) & 197.20 & 53.74 & 94.00 & 419.00 \\
Fosfatasa alcalina (UI/L) & 159.45 & 76.67 & 13.00 & 320.00 \\
GGT (UI/L) & 22.35 & 10.63 & 5.00 & 54.00 \\
\hline
\end{tabular}

aspartato aminotransferasa (AST), fosfatasa alcalina (FA) y gamma glutamil transpeptidasa (GGT) por el método UV, utilizando pruebas comerciales de Wiener Lab (EEUU). Asimismo, las globulinas se determinaron por diferencia entre los valores de proteínas totales y la albúmina. La lectura de la absorbancia se realizó en un espectofotómetro (UV Photometro 4010, Mamhein Boehringer).

Se hizo una estadística descriptiva con los valores bioquímicos hepáticos y renales, empleando la media aritmética como medida de tendencia central y la desviación estándar como medida de dispersión. Además, se presentan los valores mínimos y máximos. Se utilizó la prueba de «t» Student para determinar diferencias estadísticas entre edades (tuis y adultos).

\section{Resultados}

Los valores bioquímicos sanguíneos de la función hepática se presentan en el Cuadro 1 . Se encontró diferencia estadística en los valores de bilirrubina total e indirecta, así como en fosfatasa alcalina entre alpacas adultas y tuis, observándose mayores valores en adultos ( $p<0.05$; Cuadro 2). Asimismo, no hubo diferencia estadística por grupo etario en los demás parámetros del perfil bioquímico.

Los valores bioquímicos sanguíneos de la función renal se presentan en el Cuadro 3. No se encontró diferencia estadística en estos valores por efecto del grupo etario.

\section{Discusión}

En el Perú existen escasos estudios de bioquímica sanguínea en alpacas, de modo que la discusión comparativa se hace con los trabajos encontrados sobre el tema y con otros realizados en la llama (Lama glama), guanaco (Lama guanicoe) y vicuña (Vicugna vicugna), así como con los datos del Internacional Species Information System (1999), provenientes de exámenes médicos anuales en estas especies. 
Cuadro 2. Perfil bioquímico san guíneo hepático en alpacas adultas $(n=30)$ y tuis $(n=30)$ de la Sierra Central del Perú

\begin{tabular}{lcc}
\hline Parámetro & $\begin{array}{c}\text { Tuis } \\
(\text { media } \pm \text { d.e. })\end{array}$ & $\begin{array}{c}\text { Adultos } \\
(\text { media } \pm \text { d.e. })\end{array}$ \\
\hline Bilirrubina total $(\mathrm{mg} / \mathrm{dl})$ & $0.28 \pm 0.10^{\mathrm{a}}$ & $0.97 \pm 0.53^{\mathrm{b}}$ \\
Bilirrubina directa $(\mathrm{mg} / \mathrm{dl})$ & $0.13 \pm 0.09$ & $0.12 \pm 0.10$ \\
Bilirrubina indirecta $(\mathrm{mg} / \mathrm{dl})$ & $0.16 \pm 0.08^{\mathrm{a}}$ & $0.85 \pm 0.54^{\mathrm{b}}$ \\
Proteínas totales $(\mathrm{g} / \mathrm{dl})$ & $7.90 \pm 1.06$ & $7.55 \pm 1.28$ \\
Albúmina $(\mathrm{g} / \mathrm{dl})$ & $3.73 \pm 0.84$ & $3.53 \pm 0.36$ \\
Globulina $(\mathrm{g} / \mathrm{dl})$ & $4.16 \pm 1.40$ & $4.03 \pm 1.42$ \\
ALT $(\mathrm{UI} / \mathrm{L})$ & $23.80 \pm 11.68$ & $22.73 \pm 14.58$ \\
AST $(\mathrm{UI} / \mathrm{L})$ & $206.70 \pm 58.05$ & $187.70 \pm 48.15$ \\
Fosfatasa alcalina (UI/L) & $203.33 \pm 72.56^{\mathrm{a}}$ & $115.57 \pm 52.06^{\mathrm{b}}$ \\
GGT (UI/L) & $21.77 \pm 13.53$ & $22.93 \pm 6.80$ \\
\hline
\end{tabular}

${ }^{a, b}$ Superíndices diferentes dentro de filas indican diferencia estadística $(p \varangle 0.05)$

Cuadro 3. Valores de bioquímica sanguínea para evaluar función renal en 60 alpacas de la Sierra Central del Perú

\begin{tabular}{lccccc}
\hline \multirow{2}{*}{ Parámetro } & \multicolumn{2}{c}{ Grupo etario } & \multirow{2}{*}{ Total } & \multirow{2}{*}{ Mínimo } & \multirow{2}{*}{ Máximo } \\
\cline { 2 - 3 } & Tuis & Adultos & & & \\
\hline Urea $(\mathrm{mg} / \mathrm{dl})$ & $39.43 \pm 8.59$ & $38.77 \pm 9.57$ & $39.10 \pm 9.02$ & 20.0 & 70.0 \\
Creatinina $(\mathrm{mg} / \mathrm{dl})$ & $2.31 \pm 0.66$ & $2.11 \pm 0.36$ & $2.21 \pm 0.54$ & 1.30 & 3.70 \\
\hline
\end{tabular}

Los valores de bilirrubina total son bastante mayores a aquellos reportados por Fowler (1989) en llamas adultas $(0.1 \mathrm{mg} / \mathrm{dl})$ e ISIS (1999) para alpacas, llamas y guanacos (0.1 a $0.2 \mathrm{mg} / \mathrm{dl})$ y por Concha (2009) en vicuñas $(0.27 \mathrm{mg} / \mathrm{dl})$. Asimismo, el valor promedio de bilirrubina directa fue mayor a los reportados por ISIS (1999) en alpacas, aunque similar a los valores reportados para guanacos. También fue similar a los valores reportados por Concha (2009) en vicuñas $(0.10 \mathrm{mg} / \mathrm{dl})$. Por otro lado, los valores de bilirrubina indirecta fueron también mayores a los reportados en alpacas, llamas y guanacos $(0.1 \pm 0.1 \mathrm{mg} /)$ por ISIS (1999) y en vicuñas $(0.17 \pm 0.09 \mathrm{mg} / \mathrm{dl})$ por Concha (2009).
Las diferencias encontradas en los valores de bilirrubina entre este estudio con respecto a los otros estudios pudo deberse a las condiciones climáticas, localidad geográfica, e incluso a las diferencias propias entre laboratorios. Asimismo, drogas como antibióticos (sulfonamidas y cefalosporinas), AINES (acetaminofén y fenilbutazona), y la lipemia, hemólisis y ayuno prolongado pueden incrementar los valores de bilirrubina total en el suero (Meyer y Harvey, 1998). Según Benjamín (1991), el índice ictérico en el caballo y la vaca varía directamente con la cantidad de carotenos, carotenoides y las sustancias xantófilas en las plantas ingeridas. Por otro 
lado, la hiperbilirrubinemia está asociado al ayuno en caballos enfermos y a la anorexia en vacas (Radostitis et al., 2002).

El valor promedio de proteínas totales $(7.73 \pm 1.18 \mathrm{~g} / \mathrm{dl})$ fue ligeramente mayor a los valores reportados en alpacas por Vallenas (1957), Ellis (1982), Reynafarje et al. (1982), Quiroga (1991) e ISIS (1999), que variaron entre 5.5 y $6.79 \mathrm{~g} / \mathrm{dl}$. También fue mayor a los valores reportados en llamas por Reynafarje et al. (1982) y Garry (1989), en vicuñas por García (1988) y Van Leeuwen (1993) y en guanacos por ISIS (1999); en tanto que Concha (2009) reporta $8.27 \mathrm{~g} / \mathrm{dl}$ en vicuñas. No obstante, Benjamín (1991) considera como valores normales el rango de 5 a $8 \mathrm{~g} / \mathrm{dl}$, estando todos estos valores reportados dentro de este rango.

La albúmina es un parámetro sanguíneo que se afecta por el tipo de dieta; sin embargo, se desconoce el tipo de alimentación recibida en la mayoría de los estudios disponibles. Asimismo, el uso prolongado de una dieta baja en proteínas causaría una reducción en la concentración sérica (Meyer y Harvey, 1998). Pese a esto, el valor promedio de albúmina obtenido en el presente estu$\operatorname{dio}(3.63 \pm 0.65 \mathrm{~g} / \mathrm{dl})$ fue relativamente similar a otros valores reportados en alpacas (3.10 a $3.49 \mathrm{~g} / \mathrm{dl}$ ) por diversos autores (Vallenas, 1957; Ellis, 1982); Reynafarje et al., 1982); así como de 3.0 a $4.25 \mathrm{~g} / \mathrm{dl}$ en llamas (Reynafarje et al., 1982; Fowler, 1989; Kaneko, 1989), de 3.98 a 4.37 g/dl en vicuñas (García, 1988; Van Leeuwen, 1993; Concha, 2009), y de 3.9 en guanacos (ISIS, 1999).

Los valores de globulina presentaron una gran variabilidad $(4.10 \pm 1.40 \mathrm{~g} / \mathrm{dl})$, lo cual, si bien el promedio es mayor que en muchos estudios, es posible que se encuentre dentro del rango de varios de ellos. La literatura indica valores de 1.7 a $3.55 \mathrm{~g} / \mathrm{dl}$ en alpacas (Vallenas, 1957; Ellis, 1982; Reynafarje et al., 1982; ISIS, 1999), de 1.9 a $2.5 \mathrm{~g} / \mathrm{dl}$ en llamas (Reynafarje et al., 1982; Fowler, 1989; Garry, 1989; Kaneko, 1989), de 1.7 a 4.21 g/dl en vicuñas (García, 1988; Van Leeuwen, 1993;
Concha, 2009) y de $2.0 \mathrm{~g} / \mathrm{dl}$ en guanacos (ISIS, 1999). Los niveles de globulinas séricas se elevan en casos de enfermedades hepáticas crónicas (Longo et al., 2012), así como en casos de hemoconcentración, neoplasias, enfermedades infecciosas y una reciente exposición a antígenos (Bush, 1982). Por otro lado, disminuyen durante la última mitad de la preñez (Kaneko y Cornelius, 1971).

El valor promedio de ALT $(23.27 \pm 13.11$ UI/L), dado su amplia desviación estándar no es muy diferente de otros valores reportados en la literatura. Sin embargo, la mayoría de ellos reporta valores de 6 a 9 UI/L, tanto en alpacas (Sillau et al. 1973), llamas (Fowler, 1989; Kaneko, 1989) y vicuñas (Concha, 2009). No obstante, se encuentran valores muy diversos como promedios de $4.8 \mathrm{UI} / \mathrm{L}$ (Samaniego y Esquerre, 1978) y de 31 UI/L (Yi, 1993). Por otro lado, los valores reportados por ISIS (1999) es de $17 \pm 7,13 \pm 23$ y $16 \pm 39 \mathrm{UI} / \mathrm{L}$ para alpacas, llamas y guanacos, respectivamente.

La variabilidad de los valores de AST (197.2 $\pm 53.74 \mathrm{UI} / \mathrm{L})$ fue bastante amplia, pero con un promedio bastante mayor a aquellos de $56.74 \mathrm{UI} / \mathrm{L}$ reportado por Sillau et al. (1973), de 126.8 a 159 UI/L en alpacas jóvenes (Samaniego y Esquerre, 1978; Yi, 1993) y de 194.2 UI/L en alpacas gestantes (Yi, 1993). En llamas adultas se reportan valores entre 106 a 317 (Lassen et al., 1986; Fowler, 1989; Garry, 1989; Kaneko, 1989; ISIS, 1999) y de 116 a 165 en llamas jóvenes (Garry, 1989); mientras que en vicuñas se señalan valores de 242 a 348 UI/L (Morales, 1994; Concha; 2009) y en guanacos de $208 \pm 72$ UI/L (ISIS, 1999).

Las cantidades grandes de catecolaminas, epinefrina o norepinefrina producen marcadas elevaciones en las transaminasas séricas, lo que hay que tener en cuenta por la forma de toma de muestra en las alpacas (Highman y Altland, 1960), la cual fue por captura en tuis y antes del sacrificio en las alpacas adultas. Asimismo, Benjamín (1991) indica que la actividad disminuída de AST y 
ALT puede deberse a deficiencias de piridoxina y al uso de drogas como fenotiazina y cefazolina, mientras que su incremento puede relacionarse al efecto de corticosteroides, estrógenos, anestésicos, antibióticos (penicilina, gentamicina, lincomicina, eritromicina, sulfonamidas) y AINES (ibuprofeno y fenilbutazona), medicamentos que no fueron utilizados durante el presente estudio.

El valor promedio de fosfatasa alcalina $(159.45 \pm 76.6 \mathrm{UI} / \mathrm{L})$ fue muy superior al 23.5 y $43.6 \mathrm{UI} / \mathrm{L}$ en alpacas jóvenes reportados por Samaniego y Esquerre (1978) y Yi (1993), de $64 \pm 88 \mathrm{UI} / \mathrm{L}$ en alpacas adultas (ISIS, 1999), de 32 a 89 UI/L en llamas (Lassen et al., 1986; Fowler, 1989; Kaneko, 1989; ISIS, 1999), de 111 a 175 UI/L en vicuñas (Morales, 1994; Concha, 2009) y de $50 \pm 42 \mathrm{UI} / \mathrm{L}$ en guanacos (ISIS, 1999). Benjamín (1991) señala que los valores de FA se incrementan debido al empleo de corticosteroides y antibióticos como la eritromicina y sulfonamida, pero su uso fue descartado en los animales muestreados.

En condiciones normales, la fosfatasa alcalina alcanza mayor actividad en animales jóvenes, debido a que esta isoenzima se localiza en los osteoblastos (relacionados con la calcificación y formación de estructuras óseas) (Kaneko, 1971). También pueden elevarse por efecto del ejercicio y por el nerviosismo. Highman y Altland (1960) reportaron que grandes cantidades de catecolaminas producen marcadas elevaciones de fosfatasa alcalina.

Los valores de GGT fueron bastante variables (22.35 $\pm 10.63 \mathrm{UI} / \mathrm{L})$. Yi (1993) reporta en alpacas jóvenes y gestantes valores de 23.7 y 28.7 UI/L, respectivamente y de $23 \pm 11 \mathrm{UI} / \mathrm{L}$ en adultas (ISIS, 1999); en tanto que valores para llamas oscilan entre 15 a 32 UI/L (Lassen et al., 1986; Fowler, 1989; Garry, 1989; Kaneko, 1989; ISIS, 1999), en vicuñas de 11 a 17 UIL (Morales, 1994; Concha, 2009), y en guanacos de 31 UI/L (ISIS, 1999). Se conoce que los glucocorticoides pueden elevar el nivel de concentración de esta enzima (Merck, 2000).
El valor promedio de urea $(39.1 \pm 9.02$ $\mathrm{mg} / \mathrm{dl}$ ) fue menor al reportado por ISIS (1999), tanto para alpacas $(51.45 \pm 12.86 \mathrm{mg} / \mathrm{dl})$ como llamas $(49.31 \pm 15.00 \mathrm{mg} / \mathrm{dl})$ y guanacos $(55.74 \pm 12.86 \mathrm{mg} / \mathrm{dl})$. Los descensos en los niveles de urea son raros, aunque pueden presentarse en asociación con graves enfermedades hepáticas o malnutrición de proteínas (Sodikoff, 1996). Aún se desconocen los mecanismos de regulación responsables del incremento de la absorción renal de urea y de las variaciones de permeabilidad a la urea de la mucosa de los pre-estómagos de los camélidos (Engelhardt y Breces, 2005).

El valor promedio de creatinina $(2.21 \pm$ $0.54 \mathrm{mg} / \mathrm{dl}$ ) fue ligeramente mayor al reportado por ISIS (1999) para alpacas (2.00 \pm $0.59 \mathrm{mg} / \mathrm{dl})$, y similar para llamas $(2.29 \pm 0.70$ $\mathrm{mg} / \mathrm{dl})$ y guanacos $(2.29 \pm 0.49 \mathrm{mg} / \mathrm{dl}) . \mathrm{Al}$ igual que en el caso de la urea, una reducción en la capacidad de filtración glomerular ocasiona un aumento en los niveles séricos de creatinina. A diferencia del nitrógeno ureico sanguíneo (NUS), la creatinina no es reabsorbida por los túbulos renales (Meyer y Harvey, 1998). La creatinina sérica no es marcadamente influenciada por la dieta o hemorragia intestinal, catabolismo proteico, la edad o sexo (Benjamín, 1991; Meyer y Harvey, 1998).

\section{Conclusiones}

El efecto de la edad fue estadísticamente significativo para los valores de bilirrubina total e indirecta y fosfatasa alcalina en alpacas aparentemente normales.

\section{Literatura Citada}

1. Anderson D. 2002. Liver disease in camelids. Ohio State University, USA [Internet]. Disponible en: http:// www.rmla.com/LiverDisease.html 
2. Benjamín M. 1991. Manual de patología clínica veterinaria. México: Ed. Limusa. $421 \mathrm{p}$.

3. Bush M. 1982. Manual de laboratorio veterinario de análisis clínico. España: Ed. Acribia. 468 p.

4. Concha A. 2009. Perfil bioquímico sanguíneo hepático de vicuñas (Vicugna vicugna) criadas en cautiverio en Lima. Tesis de Médico Veterinario. Lima: Universidad Nacional Mayor de San Marcos. $84 \mathrm{p}$.

5. Daniel W. 1996. Bioestadística: bases para el análisis de las ciencias de la salud. $3^{a}$ ed. México: Ed. Limusa. 875 p.

6. Ellis J. 1982. The hematology of South American Camelidae and their role in adaptation to altitude. Vet Med Small Anim Clin 77: 1796-1802.

7. Engelhardt WV, Breces G. 2005. Fisiología veterinaria. Zaragoza, España: Ed Acribia. 683 p.

8. Fowler EM. 1989. Medicine and surgery of South American Camelids. Iowa, USA: Iowa State University Press. 391 p.

9. García E 1988. Constantes hematológicas y valores de proteínas séricas en la vicuña. Tesis de Médico Veterinario. Lima: Univ Nacional Mayor de San Marcos. 39 p.

10. Garry F. 1989. Clinical pathology of llamas. Vet Clin North Am Food Anim Pract 5: 55-70.

11. Long Dl, Faucí AS, Kasper DL, Hauser SL, Jaweson JL, Loscalzo J. 2012. Harrison. Principios de medicina interna. $18^{\mathrm{a}}$ ed. Vol II. México: McGrawHill. Interamericana. $2941 \mathrm{p}$.

12. Highman B, Atland P. 1960. Serum enzyme rise alter hipoxia and effect of autonomic blockade. Am J Physiol 199: 981-986.

13. International Species Information System (ISIS). 1999. Reference ranges for physiological data values of alpaca (Lama pacos), llama (Lama glama) and guanaco (Lama guanicoe). USA. [Internet]. Disponible en: http:// www.isis.org.com
14. Kaneko J, Cornelius C. 1971. Clinical biochemistry of domestic animals. $2^{\mathrm{a}} \mathrm{ed}$. Vol I. New York, USA: Academia Press. $439 \mathrm{p}$.

15. Kaneko J. 1989. Serum proteins and dysproteinemias. In: Kaneko JJ (ed). Clinical biochemistry of domestic animals. $4^{\text {th }}$ ed. California: Academic Press. 428 p.

16. Lassen ED, Pearson EG, Long P, Schmotzer WB, Kaneps AJ, Riebold TW. 1986. Clinical biochemical values of llamas: reference values. Am J Vet Res 47: 2278-2280.

17. Manual Merck de Veterinaria. 2000. $5^{\text {a }}$ ed. Barcelona, España: Ed Océano. $1343 \mathrm{p}$.

18. Meyer D, Harvey JW. 1998. Veterinary laboratory medicine: interpretation and diagnosis. $2^{\mathrm{a}}$ ed. USA: WB Saunders. $373 \mathrm{p}$.

19. Morales M. 1994. Algunos valores enzimáticos referenciales de vicuñas (Vicugna vicugna) criadas en cautiverio a nivel del mar en el Perú. Tesis de Médico Veterinario. Lima: Univ Nacional Mayor de San Marcos. 49 p.

20. Quiroga MX. 1991. Caracterización de algunas variables fisiológicas de alpacas criadas bajo un régimen de confinamiento en la zona central de chile. Tesis de Médico Veterinario. Santiago de Chile: Universidad de Chile. $115 \mathrm{p}$.

21. Radostitis O, Clive G, Blood D, Hinchcliff K. 2002. Enfermedades del hígado y páncreas. En: Medicina veterinaria. 9a ed. España: McGraw HillInteramericana. p 421-423.

22. Reynafarje C, Faura J, Paredes A, Villavicencio D. 1968. Erythrokinetics in high-altitude-adapted animals (llama, alpaca and vicuña). J Appl Physiol 24: 93-97.

23. Samaniego L, Esquerre CJ. 1978. Nivel plasmáticos de transaminasas: glutámico oxalacética y glutámico pirúvica en crías y alpacas adultas. Bol Soc Quím Perú 44: 148. 
24. [SENAMHI] Servicio Nacional de Meteorología e Hidrología. 2009. Boletín climático nacional enero 2009. [Internet]. Disponible en: http:// www.senamhi.gob.pe/?p=0701

25. Sillau H, Llerena L, Esquerre J, Rojas M, Alva J. 1973. Pruebas funcionales hepáticas en crías de alpacas normales e infectadas experimentalmente con Lamanema chavezi. Rev Inv Pec 2: 103- 105.

26. Sodikoff CH. 1996. Pruebas diagnósticas y de laboratorio en las enfermedades de pequeños animales. $2^{\mathrm{a}}$ ed. Madrid, España: Ed Mosby-Doyma. 405 p.
27. Vallenas A. 1957. Las proteínas totales y fraccionadas del suero sanguíneo de las alpacas. Algunas variaciones fisiológicas. Rev Fac Med Vet UNMSM 12: 40-95.

28. Van Leeuwen EMG. 1993. Perfil proteico sérico por electroforesis (PAGE) y algunas constantes fisiológicas de vicuñas en cautiverio en Perú. Tesis de Médico Veterinario. Lima: Univ Nacional Mayor de San Marcos. 83 p.

29. Yi P. 1993. Rangos referenciales de algunos valores enzimáticos séricos de alpacas jóvenes y gestantes. Tesis de Médico Veterinario. Lima: Univ Nacional Mayor de San Marcos. 32 p. 